\title{
Authentische Angst
}

\author{
Eine Skizze zum Zusammenhang von Angst, ıEigentlich- \\ keitı und Religion bei Martin Heidegger
}

Markus Höfner

\section{Authentische Angst?}

'Angst bezeichnet ein Phänomen, das sich in verschiedenen Perspektiven verstehen, erklären und mit anderen Phänomenen menschlichen Lebens in Beziehung setzen lässt. Und für viele solcher Zugänge ist es sinnvoll, Angst als Ausdruck einer abgrenzbaren Eigenschaft menschlichen (und nicht-menschlichen) Lebens $\mathrm{zu}$ thematisieren. Philosophisch (und theologisch) interessant wird das Phänomen Angst aber vor allem dann, wenn bei seiner Thematisierung unser (menschliches) Leben selbst zur Debatte steht, die Frage also wer wir sind, sein können und sein wollen. Diesem Zusammenhang zwischen dem Phänomen der Angst und der Frage nach dem >Wer menschlichen Lebens kann man im Blick auf das Leben (individueller) menschlicher Personen nachgehen oder aber ins Soziale wenden, also mit der Frage verknüpfen, wer wir sind und sein können.

Die Analyse der Angst, die Martin Heidegger in Sein und Zeit entwickelt, ist dadurch ausgezeichnet, das Phänomen der Angst und die Frage nach dem ,Wer unseres Lebens aufs Engste zusammenzurücken. In seinen Überlegungen stellt Heidegger daher nicht nur heraus, dass die Stimmung der Angst - und die affektive ‘Befindlichkeit menschlichen Lebens überhaupt - weit mehr sind als die zufällige Eigenschaft des >Vernunftwesens« Mensch. In der Angst, so Heideggers These, geht es um uns selbst, um die Frage, wie wir überhaupt ein 'Selbst sein und als wir selbst und damit reigentlich', authentisch leben können. Für die Einsichten, die Heidegger in der Ausarbeitung dieser Frage gewinnt, scheint er freilich den Preis zu bezahlen, ein authentisches Leben nur als Leben eines solitären ’Daseins` vorstellen zu können. Dass dies so ist, aber nicht so sein 
müsste, werde ich im Folgenden ${ }^{1}$ zunächst im Blick auf Sein und Zeit erläutern, indem ich den dort explizierten Zusammenhang von Angst und `Eigentlichkeit ‘ erläutere (2.). Der anschließende Rückblick auf Heideggers frühe Auseinandersetzung mit der Theologie des Paulus soll nicht nur die (`säkularisierende`) Genese tragender Grundbegriffe von Sein und Zeit (wie >Angst` und `Eigentlichkeit`) aufklären, sondern auch unterstreichen, dass die in Sein und Zeit beobachtbare Verdrängung der sozialen und politische Dimension authentischen Lebens von Heideggers eigenen Prämissen her nicht notwendig ist (3.). Schließen werde ich mit einigen Bemerkungen zu den Potentialen der Heidegger'schen Überlegungen für gegenwärtige philosophische und theologische Reflexion (4.).

\section{Authentische Angst nach Sein und Zeit}

\subsection{Die Stimmung der Angst und das menschliche In-der- Welt-sein}

Die Stimmung der Angst, so schreibt Heidegger in $\int 40$ von Sein und Zeit, »bringt das Dasein vor [...] die Eigentlichkeit seines Seins « ${ }^{2}$, vor die Möglichkeit eines authentischen Lebens. ${ }^{3}$ Es liegt auf der Hand, dass sich diese These nicht mit jedem Verständnis von Angst verträgt. Plausibel wird sie, wenn man Angst mit Heidegger als Modus menschlicher Befindlichkeit versteht und zugleich von der Emotion der Furcht abgrenzt. `Befindlichkeit` ist Heideggers Titel für die affektive Dimension menschlichen Daseins, die für menschliches Leben (`Dasein $<$ ) als In-der-Welt-sein konstitutiv ist. ${ }^{4}$ Befindlichkeit ist daher für Heidegger nicht etwas, was wir haben, sondern beschreibt, was wir sind. Und wir sind, wie der erste Abschnitt von Sein und Zeit in anti-cartesianischer Absicht unterstreicht, keine unseren Umwelten in ontologischer Selbstgenügsamkeit und reflexiver Selbsttranstransparenz gegenüberstehende `Subjekte`, sondern exis-

\footnotetext{
1 Ich greife dabei auch auf frühere Überlegungen zurück und führe sie weiter (vgl. Markus Höfner, Sinn, Symbol, Religion. Theorie des Zeichens und Phänomenologie der Religion bei Ernst Cassirer und Martin Heidegger, Tübingen 2008).

2 Martin Heidegger, Sein und Zeit [1927], Tübingen ${ }^{17}$ 1993, 188.

3 Heideggers Begriff der 'Eigentlichkeit lässt sich durchaus mit `Authentizität übersetzen, wie dies in den maßgeblichen englisch- (authenticity) und französischsprachigen (authenticité) Übersetzungen von Sein und Zeit ohnehin der Fall ist.Vgl. für notwendige Qualifizierungen unten 2.2.

4 Vgl. Heidegger, Sein und Zeit, \29.
} 
tieren immer schon in solchen Umwelten. Wir müssen und können so, als >In-der-Welt-sein<, existieren, weil wir in der Praxis unseres Lebens auf diese Umwelten angewiesen und uns diese als Horizont einer für unseren Lebensvollzug relevanten (’bedeutsamen`) 'Welt erschlossen sind. Und nur in der Erschlossenheit dieses Relevanzhorizonts können - und müssen - wir uns in unserem Lebensvollzug zu anderem (sinnerweltlichen Seienden`) und anderen (`Mit-Dasein $\triangleleft)$ verhalten. An dieser Erschlossenheit lassen sich nach Heidegger nun zwei Dimensionen unterscheiden, das Verstehen ${ }^{5}$ als kognitive und eben die Befindlichkeit als affektive Zugänglichkeit einer `bedeutsamen Welt. $^{6}$

Indem Heidegger die Befindlichkeit als Dimension der Erschlossenheit fasst, unterstreicht er nicht nur, dass Affektivität eine integrale Auszeichnung menschlichen Lebens darstellt (und nicht die zufällige Eigenschaft des >Vernunftwesens` Mensch) und sich als Modus unseres `Da-seins in einer > Welt nicht auf sinnere psychische Zustände reduzieren lässt. Weil sich Befindlichkeit und Verstehen als Dimensionen der Erschlossenheit nicht trennen lassen, gilt aus seiner Sicht auch, dass menschliches Leben immer durch ein Zugleich von Affektivität und Kognition bestimmt ist. Ein reines<, affektloses Verstehen ist daher für Heidegger ebenso eine Fehlabstraktion wie eine sreines, kognitionslose Befindlichkeit. ${ }^{7}$

Wenn Heidegger nun die Angst vor diesem Hintergrund als einen Modus der Befindlichkeit beschreibt, so nimmt er dabei eine Differenz zwischen (gegenstandslosen) Stimmungen und (gegenstandsbezogenen) Emotionen oder Affekten in Anspruch. Affekte sind, wie Heidegger am Beispiel der Furcht erläutert, ${ }^{8}$ intrinsisch mit einem intentionalen `Gegenstand ‘ verbunden: >Furcht ‘ gibt es nur, insofern uns im Relevanzhorizont unserer /Welt etwas (oder jemand) als bedrohlich begegnet und wir uns im Fürchten darauf beziehen. ${ }^{9}$ Und

\footnotetext{
5 Vgl. ebd., SS 31-33.

${ }^{6}$ Die in Sein und Zeit $₫ 34$ verhandelte $>$ Rede $(\lambda o ́ \gamma o \varsigma)$ bezeichnet keine dritte Dimension der Erschlossenheit, sondern die gliedernde Artikulation der in Verstehen und Befindlichkeit erschlossenen `Bedeutsamkeit der Welt, die in der Vielfalt konkreter Bedeutungen die Einheit des menschlicher Erfahrung zugänglichen Sinns (von 'Sein`) gewährleistet.

7 Was für Heidegger freilich - anders als für manche aktuellen Emotionstheorien nicht heißt, Affektivität und Affekte als Kognitionen zu denken.

8 Vgl. Heidegger, Sein und Zeit, $\int S 30+68$ b.

9 Ausschließen will Heidegger dabei die Vorstellung, der `Gegenstand der Furcht oder anderer Affekte würde zunächst semotionslos konstatiert, um ihm dann in einem (Wert)Urteil den Charakter des Bedrohlichen (oder andere affektive Qualitäten) zuzulegen.
} 
dieses Sich-Fürchten impliziert für Heidegger, dass der >Gegenstand der Furcht für uns bedrohlich ist: Worum es in der Furcht (und anderen Affekten) geht, ist der menschliche Lebensvollzug selbst, für dessen Möglichkeiten etwas oder jemand als abträglich (zuträglich etc.) erfahren wird. Es ist dieses /Worum<, das für Heidegger die Affekte mit den Stimmungen verbindet und beide als Ausdruck derjenigen Befindlichkeit ausweist, die wir in unserem In-der-Welt-sein sind. Im Unterschied zu den Affekten sind Stimmungen jedoch aus Heideggers Sicht nicht auf etwas oder jemand im Relevanzhorizont unserer Welt bezogen, sondern auf das menschliche In-der-Weltsein selbst. Exemplarisch zeigt sich dies nach Heidegger in der Stimmung der Angst.

Denn die Angst, so hebt Heidegger hervor, ${ }^{10}$ unterscheidet sich vom Affekt der Furcht durch ihre Unbestimmtheit. Weil in der Angst alle in ihrer Wirklichkeit bestimmten `Gegenstände menschlichen Verhaltens irrelevant werden - weshalb wir nach Heidegger vor ınichts` Angst haben ${ }^{11}$-, rückt der Horizont einer für uns `bedeutsamen Welt selbst in den Fokus. Dieser aber liegt als unbestimmter Horizont des Möglichen nicht nur allem (intentionalen) Verhalten $\mathrm{zu}$ etwas oder jemand voraus, sondern ist integrales Moment menschlichen Lebensvollzugs als In-der-Welt-sein und seine Unbestimmtheit daher Ausdruck für dessen Möglichkeitscharakter als ১Seinkönnen . Unser Leben als `Seinkönnen « ist deshalb für Heidegger nicht nur - wie im Affekt der Furcht - das, sworum < wir, sondern zugleich das, swovor < wir Angst haben. Gerade in ihrer Unbestimmtheit ist die Angst daher nach Heideggers Analyse selbstbezüglich und konfrontiert uns mit der `Unheimlichkeit` unseres eigenen Lebens, dessen kontingenter Vollzug als ıSeinkönnen uns als denen, die wir faktisch sind, unausweichlich bevorsteht.

Als vorphilosophisches (>ontisches`) Phänomen bietet die Stimmung der Angst daher aus Heideggers Sicht einen ausgezeichneten Zugang zum menschlichen In-der-Welt-sein, der sich philosophisch im Sinne einer phänomenologischen Ontologie explizieren lässt. ${ }^{12}$ Nachdem die einzelnen Aspekte menschlichen In-der-Welt-seins

\footnotetext{
${ }^{10} \mathrm{Vgl}$. Heidegger, Sein und Zeit, $\$ \int 40+68 \mathrm{~b}$.

11 Wenn auch nicht vor dem Nichts, wie es wenig später bei Heidegger heißt (vgl. Martin Heidegger, Was ist Metaphysik? [1929], in: Ders., Wegmarken, Frankfurt a.M. 1967, $\left.{ }^{3} 1996,103-123\right)$.

${ }^{12}$ Heideggers Fokussierung auf Angst (und Furcht) hat daher methodischen Sinn und ist nicht Ausweis eines 'pessimistischen Menschenbildes. Das bei Heidegger prominenteste andere Beispiel einer solchen das In-der-Welt-sein erschließenden Stimmung ist die Langeweile (vgl. Martin Heidegger, Die Grundbegriffe der Metaphysik. Welt-Endlichkeit-Einsamkeit [Gesamtausgabe 29/30], Frankfurt a.M. 1983, 89-249).
} 
bereits Thema der vorhergehenden Paragraphen von Sein und Zeit waren, lässt sich dieses nach Heidegger im Ausgang vom Phänomen der Angst als eine einheitliche Struktur verstehen und mit dem Ausdruck der Sorge auf den Begriff bringen:Als Selbstzweck ('Sorge< um sich) ist menschliches Leben dasjenige, worauf alle Mittel-ZweckRelationen in der Praxis menschlichen Verhaltens (’Besorgen $\triangleleft$ ) letztlich zurückgehen. Und weil ১Sorger für Heidegger den Vollzug menschlichen Lebens beschreibt, muss diese Struktur des In-derWelt-seins aus seiner Sicht zeitlich, als Ausdruck der Zeitlichkeit menschlichen Lebens, verstanden werden. ${ }^{13}$ In dieser Zeitlichkeit ist menschliches In-der-Welt-sein daher für Heidegger als eine "Strukturganzheit $\ll^{14} \mathrm{zu}$ beschreiben, die drei sgleichursprünglicher Momente umfasst: Als `Seinkönnen bezieht sich menschliches Leben auf bevorstehende Möglichkeiten seines Vollzugs (Existenzialität oder Entwurf; Zeitmodus der Zukunft), befindet sich dabei immer schon als das, was es ist, in spezifischen Kontexten (Faktizität oder Geworfenheit; Zeitmodus der Vergangenheit) und verhält sich so zu anderen und anderem (Verfallen; Zeitmodus der Gegenwart). ${ }^{15}$

So sehr nun die (vorphilosophische) Stimmung der Angst einen philosophischen Zugang zu dieser Strukturganzheit der Sorge ermöglicht, wird in ihr Heidegger zufolge doch in besondere Weise das Moment der Faktizität oder Geworfenheit akzentuiert. ${ }^{16}$ Das -Wovor der Angst ist daher nach Heidegger präzise zu bestimmen als das "pure Daß " $^{17}$ unseres Lebens, die Tatsache also, dass wir im Vollzug unseres Lebens immer vom faktischen, nicht selbst-gewählten hier und jetzt unserer eigenen Existenz ausgehen müssen. In der Angst erfahren wir uns als die, die wir immer schon gewesen sind (Zeitmodus der Vergangenheit), weshalb sich ihre Pointe auch in Gestalt eines Seufzers ausdrücken lässt: »Well, I guess we'll just have

\footnotetext{
${ }^{13}$ Heideggers Angst-Analyse und die an sie anschließende Explikation der SorgeStruktur bilden daher das Scharnier zwischen der vorbereitenden Fundamentalanalyse des Daseins im ersten und der Interpretation des Zusammenhangs von 'Dasein und Zeitlichkeit im zweiten (veröffentlichten) Teil von Sein und Zeit.

${ }^{14}$ Heidegger, Sein und Zeit, 193.

${ }^{15}$ In Heideggers Worten: Die Sorge als "formal existenziale Ganzheit des ontologischen Strukturganzen des Daseins" ist zu beschreiben als "Sich-vorweg-schon-seinin-(der-Welt-) als Sein-bei (innerweltlich begegnenden Seienden). (Ebd., 192).

${ }^{16}$ Diese Akzentuierung gilt nach Heidegger für alle Modi der Befindlichkeit: In unserer Affektivität ist uns erschlossen, dass und wie wir uns immer schon als die, die wir sind, in spezifischen Kontexten >be-finden<.

${ }^{17}$ Heidegger, Sein und Zeit, 343.
} 
to go on from here. ${ }^{18}$ Dieses >pure $\mathrm{Da} ß$ unseres Lebens ist dabei für Heidegger allerdings kein mit anderen geteiltes, sondern muss individuell gedacht werden: Angst haben wir nach Heideggers Analyse um und vor unserem je eigenen Leben in seiner Singularität. Als selbstbezügliche Stimmung ist die Angst daher asozial, sie svereinzelt und konfrontiert uns so mit der Unvertretbarkeit unseres Lebens in seiner kontingenten Faktizität. Und gerade darin bringt die Stimmung der Angst nach Heideggers Analyse menschliches Dasein vor "die Eigentlichkeit seines Seins" ${ }^{19}$

\subsection{Authentisch leben: Angst und >Eigentlichkeitı}

'Eigentlichkeit bezeichnet für Heidegger eine Art und Weise des Lebensvollzugs, in der wir uns selbst >zu eigen sind. 'Eigentlich oder authentisch zu leben heißt daher Heidegger zufolge nicht, unser (irgendwie schon >gegebenes`) reigentliches Selbst im Vollzug unseres Lebens zum Ausdruck zu bringen. ${ }^{20}$ Vielmehr ist für Heidegger dann von einem authentischen 'Wier menschlichen Lebens zu reden, wenn wir dieses selbstständig und selbstbestimmt vollziehen. Selbständig und selbstbestimmt aber ist unser Lebensvollzug, wenn wir uns die Möglichkeiten unseres Lebens nicht von anderen und anderem vorgeben lassen, sondern sie von dem singulären sSeinkönnen her bestimmen, das wir faktisch sind. Ein authentisches Leben, in dem wir uns selbst `zu eigen sind, ist daher für Heidegger ein Leben aus eigener Freiheit, die als Freiheit menschlichen In-der-Welt-seins nicht souverän ist, sondern situativ, als selbstbestimmter Umgang mit faktischen Lebensmöglichkeiten gedacht werden muss. ${ }^{21}$

Heideggers These, dass die Möglichkeit eines solchen authentischen Lebensvollzugs durch die Stimmung der Angst allererst eröffnet wird, macht natürlich nur Sinn, wenn >Eigentlichkeit< nicht die default-Position menschlichen Lebens ist, von der man selbstverständlich ausgehen könnte. Und in der Tat vollzieht sich menschliches Leben nach Heideggers Diagnose szunächst und zu-

\footnotetext{
${ }^{18}$ John Haugeland, Dasein Disclosed. John Haugeland's Heidegger, Cambridge, MA 2013, 234.

${ }^{19}$ Heidegger, Sein und Zeit, 188; meine Herv.

${ }^{20}$ Dies unterscheidet Heideggers Konzept der 'Eigentlichkeit von einem expressivistischen Verständnis von Authentizität, wie es etwa Charles Taylor ausgearbeitet hat (vgl. Charles Taylor, The Ethics of Authenitictiy, Cambridge, MA 1991).

${ }^{21}$ Heideggers >Phänomenologie der Freiheit verbindet somit die Dimension der Willens- oder Entscheidungsfreiheit (Selbstbestimmung) mit der der auf faktische Möglichkeiten bezogenen Handlungsfreiheit. Vgl. Günter Figal, Martin Heidegger. Phänomenologie der Freiheit, Frankfurt a.M. 1988, v.a. 98-133.
} 
meist nicht authentisch, sondern im Modus der (Uneigentlichkeit: Wir leben Heidegger zufolge immer schon so, dass wir uns nicht zzu eigen sind. ${ }^{22}$ Vielmehr lassen wir uns die Möglichkeiten unseres eigenen Lebens von anderem und anderen vorgeben, indem wir uns in unserem Verhalten zu >Dingen in ein verdinglichtes Selbstmissverständnis verstricken (statt unser `Selbst` als zeitlichen Vollzug zu begreifen) und in unserem Zusammensein mit anderen Menschen den Vollzug unseres Lebens einem anonymen ‘Man nivellierenden und normalisierenden `Gerede `Uneigentlichkeit` wird die Erschlossenheit menschlichen In-derWelt-seins somit durch nicht-selbstgewählte Bestimmungen vverschlossen Befindlichkeit: Das anonyme `Man « "braucht Stimmung und `macht sie für sich ${ }^{23}$, wie Heidegger unter Verweis auf die Rhetorik des Aristoteles hervorhebt. Nach Heideggers Analyse ist menschliches In-der-Welt-sein ein unhintergehbar soziales Phänomen - das (kooperative) $>$ Mit-Sein $<$ mit anderen gilt Heidegger ebenso als $>$ Existenzial wie das (gesellschaftliche) >Man einer immer schon durch soziale Normen artikulierten, öffentlichen $>$ Welt.${ }^{24}$ Zugleich jedoch steht diese sneutrale< Sozialität nach seiner Darstellung immer schon unter der Herrschaft der `Uneigentlichkeit`, die uns an andere und anderes sverfallen lässt und so den Selbstverlust zum Normalzustand menschlichen Lebens macht. ${ }^{25}$

Vor dem Hintergrund dieser ınormalen Uneigentlichkeit menschlichen Lebens nun lässt sich die Möglichkeit authentischen Lebensvollzugs Heidegger zufolge nicht als Leistung bewusster Selbstreflexion verständlich machen. Denn dies würde ein selbstbewusstes sSubjekt< schon voraussetzen, dessen Möglichkeit es nach Heidegger gerade aufzuklären gilt. Ein reigentlicher Lebensvollzug muss daher aus Heideggers Sicht als Ergebnis einer Befreiung aus dem Selbstmissverständnis uneigentlichen Lebens gedacht werden, die sich als ,Umkehr` oder Konversion beschreiben lässt und eines unverfügbaren,

\footnotetext{
${ }^{22}$ Vgl. Heidegger, Sein und Zeit, $\int S$ 25-27.

${ }^{23}$ Ebd., 138.

${ }^{24}$ Zur Dimension dieser sneutralen Sozialität in $\$ \int 5$ 25-27 von Sein und Zeit vgl. Hubert L. Dreyfus, Being-in-the-world. A Commentary on Heidegger's 'Being and Times, Division I, Cambridge, MA 1991.

${ }^{25}$ Vgl. zu dieser Ambivalenz der Sozialität in Sein und Zeit Hans Bernard Schmid, Gemeinsames Dasein und die Uneigentlichkeit von Individualität. Elemente einer nicht-individualistischen Interpretation des Daseins, Deutsche Zeitschrift für Philosophie 49 (2001), 665-684.
} 
gleichsam vvon außen kommenden Anstoßes bedarf. ${ }^{26}$ Eben einen solchen Anstoß aber bietet nach Heidegger die Stimmung der Angst, die menschliches Leben unwillkürlich süberfällt‘, auf seine kontingente Faktizität vereinzelt und sich darin als seigentlicher Modus menschlicher Befindlichkeit erweist. Sie verbindet sich dabei, wie die anschließenden Analysen in Sein und Zeit zu zeigen versuchen, ${ }^{27}$ mit dem >Vorlaufen in den eigenen Tod als dem seigentlichen Modus des Verstehens, in dem sich menschliches Leben als endliches und gerade darin unvermeidliches `Seinkönnen ‘ zeigt, und mit dem sschweigenden Ruf des Gewissens, der als reigentlicher Modus der Rede das nivellierende 'Gereder unterbricht und dadurch von der Herrschaft des `Man des eigenen Selbst aufruft. ${ }^{28}$ Und wenn wir dieser (alternativlosen) Wahl nicht ausweichen, leben wir rentschlossen<, und das heißt: aufgeschlossen für die Unbestimmtheit unseres 'Seinkönnens`, die es in eigener Freiheit zu bestimmen gilt. Vor diesem Hintergrund lässt sich ein reigentlicher Lebensvollzug nach Heidegger als spezifische Art und Weise beschreiben, das eigene In-der-Welt-sein in der Strukturganzheit der Sorge zeitlich zu vollziehen. Als singuläres `Seinkönnen` leben wir >eigentlich`, insofern wir aus dem (selbstbestimmten) Entwurf eines für uns guten Lebens `Zukunft auf unsere faktischen Möglichkeiten >Vergangenheit` zurückkommen und diese in unserem Verhalten zu anderen und anderem vollziehen 'Gegenwart.$^{29}$

Wie nun die Angst nach Heideggers Analyse eine unbestimmte und gerade darin selbstbezügliche Stimmung ist, so bleibt auch sein Konzept authentischen Lebensvollzugs gänzlich formal. Liest man Heideggers Konzeption der 'Eigentlichkeit< als Version einer Ethik des Guten, so lässt sich dieser formale Charakter als Ausdruck ihrer Modernität verstehen. ${ }^{30}$ Zugleich jedoch liegt in dieser Formalität reigentlichen Lebens ein Hinweis auf die Probleme der Heidegger'schen Konzeption. Diese bestehen freilich nicht darin, dass Heidegger zu einem authentischen Leben als Alternative zur normalen `Uneigentlichkeit` aufrufen würde - die Alternative einer

\footnotetext{
${ }^{26}$ Vgl. Barbara Merker, Selbsttäuschung und Selbsterkenntnis. Zu Heideggers Transformation der Phänomenologie Husserls, Frankfurt a.M. 1988, 153-193.

${ }^{27} \mathrm{Vgl}$. Heidegger, Sein und Zeit, v.a. $\int S 50-53,56-60$.

${ }^{28}$ Zum Motiv der Selbstwahl vgl. Ernst Tugendhat, Selbstbewußtsein und Selbstbestimmung. Sprachanalytische Interpretationen, Frankfurt a.M. 1979, 225-244.

${ }^{29}$ Vgl. Heidegger, Sein und Zeit, SS $64 f$.

${ }^{30}$ Vgl. Martin Seel, Heidegger und die Ethik des Spiels, in: Forum für Philosophie Bad Homburg (Hg.), Martin Heidegger. Innen- und Außenansichten, Frankfurt 1989, 244-272.
} 
individuellen Sonderexistenz, die sich von jeder Bindung an andere und anderes lösen würde und in der die Stimmung der Angst auf Dauer gestellt wäre. Dies wäre vielmehr ein existenzialistisches Missverständnis der Heidegger'schen Überlegungen, mit dem die für Sein und Zeit tragende Differenz von existenziellen Beispielphänomenen und existenzialer, philosophischer Explikation menschlichen In-der-Welt-seins ebenso unterlaufen wird wie die Einbettung auch noch dieser existenzialen Analyse in die bedeutungstheoretische Frage nach dem Sinn von Sein. ${ }^{31}$ Einem solchen Missverständnis gegenüber ist es für Heidegger klar, dass zwar die Möglichkeit der 'Eigentlichkeit durch existenzielle Phänomene wie die svereinzelnder Stimmung der Angst eröffnet werden muss, dass jedoch der zeitliche Vollzug eines authentischen Lebens nur als »modifiziertes Ergreifen ${ }^{32}$ der normalen `Uneigentlichkeit vorstellbar ist. ${ }^{33}$ Denn als In-der-Welt-sein befinden wir uns immer schon in Bezügen zu anderen und anderem und damit zugleich in einer durch soziale Normen artikulierten, öffentlichen /Welt‘, mit der uns faktische Lebensmöglichkeiten vorgegeben sind. Ein authentischer Lebensvollzug kann daher nicht in einer solitären Existenz, sondern nur darin bestehen, dass wir uns solche faktischen Lebensmöglichkeiten aus eigener Freiheit aneignen - was eine kritische Auswahl nicht aus-, sondern einschließt. ${ }^{34}$ So sehr jedoch ein solches Verständnis authentischen Lebens auf der Linie von Heideggers Artikulation der vorgängigen Einbettung menschlichen In-der-Welt-seins in natürliche und soziale Kontexte liegt, so wenig gelingt es ihm, authentisches Leben als >modifiziertes Ergreifen der faktischen Lebensmöglichkeiten einer gemeinsamen Welt verständlich zu machen. Denn dazu müssten soziale Formen authentischen Lebens in den Blick genommen werden, wie sie als Resultat solcher reigentlicher Modifikation faktischer Lebensmöglichkeiten einer gemeinsamen Welt erwartbar sind. ${ }^{35} \mathrm{Im}$ Blick auf kooperative Beziehungen bietet Heidegger dazu

\footnotetext{
${ }^{31}$ Ein solches existenzialistisches Missverständnis von Sein und Zeit lässt den affirmativen Anschluss an Heidegger (Satre) ebenso zu wie die kritische Abgrenzung (Adorno).

32 Heidegger, Sein und Zeit, 179.

${ }^{33}$ Und nicht als ein "vom Man abgelöste[r] Ausnahmezustand des Subjekts" (Ebd., 130).

${ }^{34}$ Diese kritische Pointe der des Konzepts der `Eigentlichkeit` gibt man preis, wenn man allein auf Heideggers soziale Kontextualisierung des menschlichen In-der-Weltseins abstellt, wie dies in spragmatistischen Heidegger-Lektüren oft der Fall ist.

${ }^{35}$ Vgl. etwa die - mit und gegen Heideggers Analyse des `Man zeption authentischer (politischer) Öffentlichkeit bei Hannah Arendt,Vita activa oder Vom tätigen Leben, München 1967, \ 24 (213-222).
} 
in Sein und Zeit jedoch nur wenige Hinweise zur >Fürsorge als authentischer Form des Verhaltens zu anderen, ${ }^{36}$ während er im Blick auf gesellschaftliche, durch soziale Normen koordinierte Sozialität auf die kollektivistische Figur des Volkes ausweicht ${ }^{37}$. Spätestens an diesem Punkt jedoch zeigt sich, dass Heidegger Authentizität in Sein und Zeit nicht nur formal, sondern monologisch, als einen exklusiven Selbstbezug denkt, der sich zwar vom einzelnen Individuum auf ein Kollektiv übertragen lässt, der aber den Weg zu koordinierten Formen von Sozialität und zu einem aus eigener Freiheit >modifizierten Ergreifen faktischer Lebensmöglichkeiten einer gemeinsamen Welt versperrt. Und dieses Problem einer monologisch gedachten >Eigentlichkeit wird in Sein und Zeit durch Heideggers Tendenz verfestigt, dem solitären Dasein seiner vorgängigen Kontextualisierung zum Trotz einen souveränen Welt-Entwurf zuzuschreiben und das Projekt einer Fundamentalontologie damit an einen "HyperCartesianismus ${ }^{38}$ zurückzubinden. ${ }^{39}$ Die unbestimmte und selbstbezügliche Stimmung der Angst, die nach Heideggers Analyse doch die Faktizität (und damit Kontextualität) des >vereinzelten S Selbst erschließen soll, verweist daher letztlich auf dieses als den einen Ursprung, der der Struktur des In-der-Welt-seins noch vorausliegt. ${ }^{40}$

\footnotetext{
${ }^{36} \mathrm{Vgl}$. Heidegger, Sein und Zeit, $\ 26$. Solche `Fürsorge < ist bei Heidegger allerdings nicht auf Gegenseitigkeit angelegt, sondern wird vom Verhalten je individuellen authentischen Lebens her gedacht.

${ }^{37}$ Vgl. Ebd., \ 74 und Heidegger, Die Selbstbehauptung der deutschen Universität [1933], in: Ders., Reden und andere Zeugnisse eines Lebensweges (Gesamtausgabe 16), Frankfurt 2000, 107-117.

${ }^{38}$ Thomas Rentsch, Negativität und praktische Vernunft, Frankfurt 2000, 39.

${ }^{39}$ Dies hat auch für das fundamentalontologische Projekt von Sein und Zeit desaströse Folgen, weil Heidegger auf dieser Grundlage den Sinn von Sein an die >Horizonter der Zeitlichkeit zurückbindet, in denen sich das welt-konstitutive Dasein Sein repräsentiert - was auf eine repräsentationalistische Bedeutungstheorie zurückführt, wie sie Heidegger eigentlich überwinden wollte.

${ }^{40}$ Die Spuren dieser (neukantianischen) /Logik des Ursprungs zeigen sich noch beim späten Heidegger, indem nun das Sein statt des Daseins als )Ursprungく ausgezeichnet wird.
} 


\section{Die `Bedrängnisı der Parusie und das authen- tische Leben im Glauben: Zu Heideggers früher Paulus-Lektüre}

Heideggers Analyse der Angst und ihrer Bedeutung für ein authentisches Leben in Sein und Zeit schöpft aus vielen Quellen. In einer Fußnote verweist Heidegger selbst auf die "christliche Theologie» und nennt Augustins Konzept des timor castus, Luthers Kommentar zum Buch Genesis und Kierkegaards Der Begriff Angst. ${ }^{41}$ Eine Quelle, die Heidegger nicht nennt, ist seine Auseinandersetzung mit der Theologie des Paulus in der frühen Freiburger Vorlesung Einleitung in die Phänomenologie der Religion (1920/21) ${ }^{42}$ Diese Paulus-Lektüren sind jedoch für das Verständnis der späteren Überlegungen in Sein und Zeit - und im Blick auf gegenwärtige Möglichkeiten ihrer philosophischen und theologischen Rezeption - besonders instruktiv. Als rreligionsphilosophisch können diese frühen Überlegungen dabei nur mit Einschränkungen gelten - jedenfalls dann, wenn man Religionsphilosophie als kritische Hermeneutik religiöser Lebensformen versteht, die deren interne Rationalität in philosophischem Horizont erkundet. Denn Heideggers Paulus-Lektüre dokumentiert nicht nur sein Ringen mit der eigenen theologischen Herkunft, das ihn mitunter als Theologen sprechen lässt. ${ }^{43}$ Vor allem aber geht es ihm als Philosoph darum, das urchristliche Leben, wie es in den paulinischen Texten zugänglich wird, gerade nicht als spezifisch religiöse Lebensform zu analysieren, sondern als Paradigma menschlichen Lebens überhaupt auszuweisen. Dabei kommt es aus Heideggers Sicht darauf an, das Leben der ersten Christen nicht als historische >Gegebenheit zu verstehen, die sich als Objekt historischer Erkenntnis fassen ließe. ${ }^{44}$ Ziel einer Phänomenologie der Religion muss es nach Heideggers Überzeugung vielmehr sein, das urchristliche Leben in seinem Vollzug zu explizieren. Und eben dazu bieten Briefe des Paulus nach Heidegger einen ausgezeichneten Zugang, weil diese

\footnotetext{
${ }^{41}$ Heidegger, Sein und Zeit, 190, Anm. 1.

${ }^{42}$ Martin Heidegger, Einleitung in die Phänomenologie der Religion [1920/21], in: Ders., Phänomenologie des religiösen Lebens (Gesamtausgabe 60), Frankfurt a.M. 1995, 1-156.Vgl. zur Interpretation auch Dominic Kaegi, Die Religion in den Grenzen der bloßen Existenz. Heideggers religionsphilosophische Vorlesungen von 1920/21, Internationale Zeitschrift für Philosophie 1 (1996), 133-149.

${ }^{43}$ Unter dem Einfluss von Luther, Kierkegaard und Overbeck zielen Heideggers Überlegungen auch auf eine Überwindung der seine theologischen Studienjahre prägenden neu-scholastischen Metaphysik.

${ }_{44}$ Wie dies nach Heidegger etwa in Ernst Troeltschs Religionsphilosophie geschieht.
} 
einen genuinen Ausdruck "urchristliche[r] Lebenserfahrung «" ${ }^{45}$ darstellen, der noch nicht durch die objektivierenden und platonisierenden Tendenzen späterer Theologie- und Philosophiegeschichte verfälscht ist. ${ }^{46} \mathrm{Um}$ sicherzustellen, dass sich die philosophische Explikation dieser Briefe nicht an isolierten Inhalten urchristlicher Lebenserfahrung festmacht, sondern auf deren Vollzug fokussiert bleibt, nimmt Heidegger das wesentliche Ergebnis seiner Analysen im Sinne einer sformalen Anzeige vorweg: »Die christliche Religiosität lebt die Zeitlichkeit als solche. ${ }^{47}$ Wie ist dies zu verstehen?

(Ur)christliches Leben, so hebt Heidegger in seiner Exegese des 1. Thessalonicherbriefs hervor ${ }^{48}$, vollzieht sich in Erwartung der $\mathrm{Pa}$ rusie, der eschatologischen Wiederkunft Christi, und darin in einer

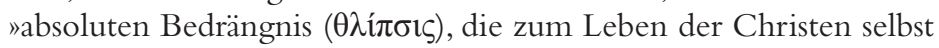
gehört. « ${ }^{49}>$ Bedrängend ist die Parusie Christi nach Heideggers Analyse allerdings nicht als das kommende Ereignis einer >objektiven Zukunft. Ein Leben im Glauben würde sich in dieser Perspektive durch den Glauben an das zukünftige Ereignis der Parusie (und andere spezifisch christliche Inhalte) auszeichnen, und die zum christlichen Leben gehörende >Bedrängnis` wäre dann als Folge dieses Glaubens zu beschreiben. Nach Heideggers Interpretation jedoch ist christlicher Glaube (allein) als eine bestimmte Art und Weise des Lebensvollzugs zu verstehen und die urchristliche Erwartung der Parusie daher als Ausdruck der Einsicht, dass dieser Lebensvollzug eine `Bedrängnis` ist. Und eine `Bedrängnis` ist das Leben im Glauben, weil man es nur haben kann, indem man es unausweichlich immer neu vollzieht. Aus diesem Grund, so Heidegger, weist Paulus die Frage der Thessalonicher nach dem 'Wann<, dem kommenden Zeitpunkt der Parusie entschieden ab und verweist sie stattdessen zurück auf den gegenwärtigen Vollzug ihres Lebens im »Wie der christlichen Lebenshaltung ${ }^{5} .{ }^{50}$ Und genau dieses $>$ Wie< christlichen Lebens ist nach Heideggers Interpretation das, was die Christen in

\footnotetext{
${ }^{45}$ Heidegger, Einleitung, 80; meine Herv.

${ }^{46}$ Die Paulus-Briefe bilden daher für Heidegger den geeigneten Ausgangspunkt für die "Destruktion der christlichen Theologie und der abendländischen Philosophie» (Ebd., 135), deren Ontologie der Vorhandenheit es aus seiner Sicht phänomenologisch zu überwinden gilt.

${ }^{47}$ Heidegger, Einleitung, 80; meine Herv.

${ }^{48}$ Neben diesem werden der Galaterbrief und der (pseudo-paulinische) 2. Thessalonicherbrief Gegenstand der Heidegger'schen Analyse.

${ }^{49}$ Heidegger, Einleitung, 97.

${ }^{50}$ Ebd., 95; meine Herv. "Das Wann ist bestimmt durch das Wie des Sich-Verhaltens, dies ist bestimmt durch den Vollzug der faktischen Lebenserfahrung in jedem ihrer Momente« (Ebd., 106).
} 
Thessaloniki mit der Verkündigung des Paulus angenommen haben - weshalb sie Paulus in seinem Brief nicht nur auf die Erwartung der Parusie, sondern zugleich auf ihr `Gewordensein « verweist. Auch dieses `Gewordensein « ist dabei Heidegger zufolge kein Ereignis einer sobjektiven Vergangenheit, sondern bestimmt als Annahme der Art und Weise christlichen Lebens dessen gegenwärtigen Vollzug. Dass die urchristliche Lebenserfahrung sdie Zeitlichkeit als solcher lebt heißt daher für Heidegger, dass im Horizont der Parusie ('Zukunft) die durch die paulinische Verkündigung eröffnete Möglichkeit der christlichen Lebensweise (>Vergangenheit) je aktuell ergriffen und gelebt wird ( $>$ Gegenwart ). Dass sich christliches Leben nicht (nur) in der Zeit, sondern als Zeitlichkeit vollzieht und nur in diesem unausweichlichen Vollzug sgehabt werden kann, macht seine Ungesichertheit aus, wie sie in der (Befindlichkeit der) >Bedrängnis erschlossen ist. Zugleich jedoch ist dieses zeitliche $>$ Wier christlichen Lebensvollzugs nach Heideggers Interpretation durch ein "glaubendes Wissen ${ }^{51}$ ausgezeichnet, ${ }^{52}$ in dem die Christen in Thessaloniki sich in ihrem zeitlichen Lebensvollzug verstehen - und in diesem (angemessenen) Verständnis shaben sie sich selbst. Die urchristliche Lebenserfahrung ist daher nach Heideggers Darstellung ein authentisches Leben im Glauben.

Als authentischer Lebensvollzug steht die urchristliche Lebenserfahrung nach Heideggers Paulus-Analyse im Kontrast zu jeder Tendenz, das eigene Leben im Rekurs >objektiver Gegebenheiten zu sichern, die dem `bedrängenden Vollzug der eigenen Zeitlichkeit scheinbar enthoben sind. Im 1. Thessalonicherbrief wird diese Tendenz aus Heideggers Sicht durch diejenigen repräsentiert, die nach "Frieden und Sicherheit im faktischen Leben ${ }^{53}$ streben, indem sie 'weltlicher Inhalte ihrer Erfahrung zu einem von ihrem zeitlichen Lebensvollzug unabhängigen >Halt verabsolutieren. Und diese Sicherungsstrategie des Unglaubens betrifft auch die Erwartung der Parusie, die nun als noch ausstehendes Datum einer sobjektiven Zukunft missverstanden und daher in ihrer Bedeutung für den eigenen Lebensvollzug in seiner Zeitlichkeit verkannt wird. Gerade darin erweist sich der Unglaube Heidegger zufolge als Gestalt der Selbstentfremdung. Die ihr Leben im Unglauben leben, können

51 Ebd., 101.

${ }^{52}$ Mit diesem Ansatz beim 'glaubenden Wissen erweisen sich Heideggers PaulusAnalysen als Ausdruck des von Heidegger verfolgten phänomenologischen Programms, das er in seinen frühen Vorlesungen als `Hermeneutik der Faktizität (genitivus objectivus und subjektivus) annonciert: Als Verstehen desjenigen Verstehens, in dem sich das faktische menschliche Leben selbst versteht.

${ }^{53}$ Heidegger, Einleitung, 103 (als Auslegung von 1 Thess 5, 3). 
sich angesichts der Parusie "nicht retten, weil sie sich selbst nicht haben, weil sie das eigene Selbst vergessen haben $\aleph^{54}$. Diese Tendenz zur ungläubigen Selbstsicherung lässt sich nach Heideggers Analyse allerdings nicht auf die distinkte Gruppe der `Ungläubigen` eingrenzen, sondern gehört zum Normalzustand menschlichen Lebens. Für die Christen in Thessaloniki bildet sie daher nicht nur eine ständige Versuchung, vielmehr gehört es zu ihrem sglaubenden Wissen<, dass sie ihren authentischen Lebensvollzug nicht aus sich selbst heraus gewinnen können. Denn im Kontext der »abfallende[n] Lebenstendenz ${ }^{55}$ des Unglaubens ist ein authentisches Leben im Glauben nur möglich als Umkehr von den sgötzenhaften Sicherungsinstanzen zum (wahren) Gott ${ }^{56}$, und eine solche Umkehr wird erst möglich durch die paulinische Verkündigung der Parusie Christi: Der Glaube kommt aus dem Hören. ${ }^{57}$

An diese Skizze von Heideggers früher Paulus-Auslegung lassen sich drei Beobachtungen anschließen. Zum einen fällt auf, dass Heidegger die in den paulinischen Briefen artikulierte urchristliche Lebenserfahrung in der Tat als Paradigma faktischen menschlichen Lebens liest - wozu die spezifisch religiösen Inhalte durch Formalisierung ssäkularisiert` werden müssen. Daher beginnt Heidegger seine religionsphänomenologische Vorlesung zwar mit der - richtigen Einsicht, dass sich die inhaltlichen Bestimmungen einer Lebensform (ihr >Gehaltssinn $\prec$ und `Bezugssinn`) nur im pragmatischen Kontext ihres Vollzugs angemessen explizieren lassen (>Vollzugsssinn $ৎ) .{ }^{58}$ Indem er das Leben im Glauben dann aber als Paradigma (authentischen) menschlichen Lebens überhaupt liest, werden `Gehalts-〈 und 'Bezugssinn « christlichen Lebens zu Chiffren, die allein den formalen , Vollzugssinn $<$ menschlicher Zeitlichkeit artikulieren. Deshalb besteht die Auszeichnung authentischen Lebens im Glauben in Erwartung der Parusie Christi in der `Bedrängnis` seines Vollzugs, nicht aber in der Hoffnung auf die Gabe eschatologischer Erlösung. Die philosophische Pointe von Heideggers Paulus-Lektüre ist eine »eschatology withouth eschaton ${ }^{59}$.

Zum anderen dürfte deutlich sein, dass in Heideggers Auseinandersetzung mit Paulus zentrale Motive der Daseinsanalytik von Sein und Zeit präfiguriert werden: Die `Bedrängnis` christlichen Lebens weist

\footnotetext{
${ }^{54}$ Ebd.

${ }^{55}$ Ebd., 113.

${ }^{56}$ Ebd., 97 (als Auslegung von 1 Thess 1, 9f.).

${ }^{57}$ Vgl. ebd., 71 (in Bezug zu Gal 3, 2; Röm 10,11ff.).

${ }^{58}$ Vgl. ebd., 62-64.

${ }^{59}$ Judith Wolfe, Heidegger's Eschatology.Theological Horizons in Martin Heidegger's Early Work, Oxford 2013, 3.
} 
voraus auf die Angst, die Zeitlichkeit urchristlicher Lebenserfahrung auf die Zeitlichkeit des In-der-Welt-seins, die Unterscheidung von Glauben und Unglauben auf die Differenz von 'Eigentlichkeit< und ,Uneigentlichkeitı, die Umkehr zum Glauben auf das Konversionsgeschehen als Anfang authentischen Lebens in Sein und Zeit, die Verkündigung des Paulus als äußerer Anlass dieser Umkehr auf den sschweigenden Ruf des Gewissens. Vor diesem Hintergrund betrachtet ist die Daseinsanalytik von Sein und Zeit nicht nur, aber eben auch eine säkularisierte Version paulinischer Theologie.

Und schließlich ist auffallig, dass die Gestalt authentischen Lebens, die Heidegger in seinem philosophischen Zugriff auf Paulus gewinnt, nicht durch die monologische Zuspitzung der Eigentlichkeit geprägt ist, die seine Analysen in Sein und Zeit belastet. Die paulinische Verkündigung, wie sie in seinen Briefen sprachlich artikuliert ist, analysiert Heidegger als eine Form seigentlicher Kommunikation jenseits des Sein und Zeit prägenden Totalverdachts des `Geredes` gegenüber jeder sprachlichen Vermittlung. Und der christliche Lebensvollzug der Gemeinde in Thessaloniki erscheint in Heideggers Darstellung als eine Form authentischer Sozialität, deren `Bedrängnis` gerade nicht svereinzelt .

\section{Angst und `Eigentlichkeitı: Philosophische und theologische Perspektiven nach Heidegger}

Heideggers Überlegungen zum Zusammenhang von Angst und 'Eigentlichkeit< lassen sich philosophisch gerade dann produktiv rezipieren, wenn man sie nicht >existenzialistisch<, als >Aufruf zur Eigentlichkeit missversteht. Und ein Blick auf Heideggers frühe Paulus-Lektüren (und andere Vorlesungen aus der Vorgeschichte von Sein und Zeit) ist für eine solche Rezeption durchaus instruktiv. Denn zwar können Heideggers frühere Überlegungen in ihrem oft tentativen Charakter die systematisierten Analysen in Sein und Zeit nicht ersetzen. Wohl aber können sie deutlich machen, dass die monologische Zuspitzung der `Eigentlichkeit in Sein und Zeit kein notwendiges Implikat der Heidegger'schen >Hermeneutik der Faktizität ‘ darstellt, sondern eine problematische Verkürzung, die den überzeugenderen Aspekten Heidegger'schen Denkens zuwiderläuft. Gerade indem sie authentische Formen von (sprachlicher) Kommunikation und Sozialität in Blick nehmen, die in Sein und Zeit aus dem Blick geraten, bieten die frühen Paulus-Lektüren (und andere frühe 
Vorlesungen $^{60}$ ) (zusätzlichen) Anlass, Heideggers Verständnis von Authentizität in diese - im weiteren Sinne politische - Richtung weiterzudenken. So lässt sich etwa Heideggers Verständnis menschlicher Freiheit als kontextualisiertes und daher nicht-souveränes 'Seinkönnen zu einem Konzept sozialer Freiheit vertiefen, demzufolge die Freiheit des menschlichen Selbst nur im Miteinander mit anderen zu haben ist - und gerade deshalb als Grundlage einer reigentlichen<, in dieser Freiheit gründenden Politik zu gelten hat. ${ }^{61}$ Und erst aus der in Heideggers Angst-Analyse herausgestellten Kontingenz dieses sSeinkönnens in seiner radikalen Unbestimmtheit ergibt sich aus einer solchen Perspektive die Möglichkeit und Notwendigkeit der Gestaltung des politischen Raums, in der zwischen authentischen und nicht-authentischen Formen zu unterscheiden ist. ${ }^{62}$

Eine theologische Rezeption der Heidegger'schen Überlegungen sollte die Einsicht, dass Heidegger tragende Motive von Sein und Zeit durch die Formalisierung und Säkularisierung paulinischer Theologie gewinnt, ${ }^{63}$ als Warnung vor einer allzu direkten Adaption verstehen. Der Versuch zumal, Heideggers Daseinsanalytik aus Sein und Zeit als sneutrale - und dabei überraschend spassender - philosophische Grundlage theologischer Reflexion in Anspruch zu nehmen ${ }^{64}$ erweist sich im Licht dieser Einsicht als Selbsttäuschung, die darauf hinausläuft, Heideggers Formalisierung christlicher

\footnotetext{
${ }^{60} \mathrm{Vgl}$. zu solchen sproto-politischen Dimensionen der frühen Vorlesungen Heideggers insgesamt Ferdinando G. Menga, Ausdruck, Mitwelt, Ordnung. Zur Ursprünglichkeit einer Dimension des Politischen im Anschluss an die Philosophie des frühen Heidegger, Paderborn 2018.

${ }^{61}$ Vgl. Hannah Arendt, Freiheit und Politik, in: Dies., Zwischen Vergangenheit und Zukunft. Übungen im politischen Denken I, München 1994, 201-226. Zu einer solchen `Sozialisierung` des Heidegger'schen Ansatz würde es dann auch gehören, das Konzept der Authentizität von Heideggers Entscheidungsrhetorik zu befreien und in ein graduelles 'Mehr oder weniger zu überführen.

${ }^{62} \mathrm{Vgl}$. zu einer solchen sozialtheoretischen und politischen Lektüre der Heidegger'schen Daseinsanalytik die instruktiven Überlegungen bei Jan Slaby und Gerhard Thonhauser, Heidegger and the Affective (Un)Grounding of Politics, in: Christos Hadjioannou (Hg.), Heidegger on Affect, Cham 2019, 265-289 und Meike Siegried, Subjektivität ohne Souveränität? Politisches Denken im Ausgang von Heideggers Freiheitsbegriff(en), in: Paul Sörensen/Nikolai Münch (Hg.), Politische Theorie und das Denken Heideggers, Bielefeld 2013, 43-60.

${ }^{63}$ Schon vor der Publikation der frühen Paulus-Analysen 1995 ist dies wachen Leserinnen und Lesern natürlich nicht entgangen. Erst anhand dieser Texte lässt sich dieser Vorgang der Säkularisierung jedoch konkret nachvollziehen.

${ }^{64}$ Wie dies Heidegger jedenfalls zeitweise selbst empfehlen konnte (vgl. Martin Heidegger, Phänomenologie und Theologie [1927], in: Ders., Wegmarken, Frankfurt a.M. $\left.1967,{ }^{3} 1996,45-78\right)$.
} 
Motive theologisch wieder zu ent-formalisieren. ${ }^{65}$ Heideggers Daseinsanalytik eignet sich nicht zur philosophischen Grundlegung christlicher Theologie, die in ihrer Explikation der libertas Christiana ohnehin keine philosophische Fundierung braucht und gegen Heidegger darauf bestehen wird, dass das Selbst des Glaubens seine Freiheit extra se findet und ein Leben im Glauben daher theologisch als Gabe Gottes gedacht werden muss. In der Einsicht aber, dass solcher Glaube auch im `Mut zum (eigenen) Sein`seinen Ausdruck finden kann und sollte, ${ }^{66}$ kann sich Theologie auch von Heideggers Konzept authentischen Lebens bestärken lassen. Vor allem jedoch ist Heideggers kritische Diagnose theologisch ernst zu nehmen, dass suneigentlicher Formen menschlichen Lebens nicht zuletzt auf Strategien der Selbstsicherung beruhen, in denen menschliches seinkönnen in seiner Kontingenz verdeckt wird. Denn bereits in seiner frühen Paulus-Lektüre hebt Heidegger hervor, dass eine solche runeigentlicher Selbstsicherung auch religiöse Formen annehmen kann. Im Versuch, der `Bedrängnis` des eigenen Lebensvollzugs zu entfliehen, kann Gott zu einem höchsten Seienden vergegenständlicht und dadurch zu einem menschlich verfügbaren $>$ Halt `werden, der von der Kontingenz eigener Freiheit entlastet. ${ }^{67}$ Von dieser kritischen Einsicht, in der sich Heideggers spätere Diagnose einer sontotheo-logischen Verfassung der Metaphysik « bereits ankündigt, ${ }^{68}$ kann sich christliche Theologie daran erinnern lassen, die Gewissheit des Glaubens nicht als Sicherstellung menschlichen Lebens zu denken - und Gott nicht als höchstes Seiendes, sondern als Wirklichkeit, die menschlichem >Seinkönnen neue Möglichkeiten eröffnet, die es ohne sie nicht gäbe. $\mathrm{Zu}$ diesen Möglichkeiten würde dann nicht eine pauschale Überwindung der Angst gehören - die den Verdacht

\footnotetext{
${ }^{65}$ Vgl. John D. Caputo, Demythologizing Heidegger, Bloomington, IN 1993, 169 185.

${ }^{66}$ Vgl. Paul Tillich, The Courage to Be [1952], in: Ders., Writings on Religion / Religiöse Schriften (Main Works / Hauptwerke 5), Berlin 1988, 141-230, v.a. 221-224.

${ }^{67}$ Vgl. Heidegger, Einleitung, 122f. Geradezu konfessorisch kommentiert Heidegger: "Das ist Blasphemie! Gott ist nie ein $\gg$ Halt «." (Ebd., 122). Diese Kritik bildet dann ein Hauptmotiv von Heideggers früher Auseinandersetzung mit Augustin (vgl. Martin Heidegger, Augustinus und der Neuplatonismus [1921], in: Ders., Phänomenologie des religiösen Lebens (Gesamtausgabe 60), Frankfurt a.M. 1995, 157-299 und zur Interpretation Markus Höfner, Ist sewiges Leben inhuman? Philosophische Augustin-Lektüren bei Martin Heidegger und Paul Ricoeur, in: Günter Thomas/Markus Höfner (Hg.), Ewiges Leben. Ende oder Umbau einer Erlösungsreligion?, Tübingen 2018, 195-219, hier 196-208).

${ }^{68} \mathrm{Vgl}$. Martin Heidegger, Die onto-theo-logische Verfassung der Metaphysik [1956/57], in: Ders., Identität und Differenz (Gesamtausgabe 11), Frankfurt a.M. 2006, 51-79.
} 
einer Flucht vor der Kontingenz der Freiheit nicht zu Unrecht auf sich zöge -, wohl aber ein in Orientierung an Gott qualifizierter Umgang mit Angst und Ängsten.

- Dr. Markus Höfner ist geschäftsführender Oberassistent am Institut für Hermeneutik und Religionsphilosophie, Theologische Fakultät, Universität Zürich. Zu seinen Arbeitsgebieten gehören Religionsphilosophie und Dogmatik, insbesondere Theologie der Kirche und Theologie des Gebets. 